\title{
Temporal and spatial expression of Drosophila Neurexin during the life cycle visualized using a DNRX-Gal4/UAS-reporter
}

\author{
Mingkuan Sun*, Xiankun Zeng \& Wei Xie \\ The Key Laboratory of Developmental Genes and Human Disease, Institute of Life Sciences, Southeast University, Nanjing 210096, China
}

Received August 6, 2015; accepted August 22, 2015; published online 26 October 2015

\begin{abstract}
Drosophila neurexin (DNRX) plays a critical role in proper architecture development and synaptic function in vivo. However, the temporal and spatial expression pattern of DNRX still remains unclear. For this study, we generated a novel Drosophila transgenic strain termed the DNRX-Gal4 transgenic line, with characteristic features in agreement with the endogenous DNRX expression pattern. DNRX expression was examined by driving the expression of a GFP reporter (nuclear-localized and membrane-localized GFP) using the DNRX-Gal4 promoter. We found that DNRX was expressed preferentially in central and motor neurons in embryos, larvae and adults, but not in glial cells. DNRX was expressed in pre- and post-synaptic areas in third instar larvae neuromuscular junctions (NMJs). Reporter expression was also observed in the salivary glands, guts, wings and legs of adult flies. In the adult brain, reporter expression was observed throughout several brain regions, including the mushroom body (MBs), antennal lobe (AL) and optic lobe neurons, which is consistent with endogenous DNRX expression via antibody staining. Interestingly, DNRX was also expressed in clock neurons. Meanwhile, we found that DNRX expression in the MBs was required for olfactory learning and memory.
\end{abstract}

Drosophila, neurexin, Gal4, motor neuron, NMJs

Citation: Sun, M., Zeng, X., and Xie, W. (2016). Temporal and spatial expression of Drosophila Neurexin during the life cycle visualized using a DNRX-Gal4/UAS-reporter. Sci China Life Sci 59, 68-77, doi: 10.1007/s11427-015-4946-9

\section{INTRODUCTION}

Neurexins play an important role in connecting and maintaining neuronal synapses. Studies have indicated that vertebrate neurexins play essential roles in the development and function of synapses (Dean et al., 2003; Graf et al., 2004; Missler et al., 2003). There are three neurexin genes in mammals, each of which has two promoters, thus generating $\alpha$ - and $\beta$-neurexin (Missler et al., 1998; Tabuchi and Sudhof, 2002). In Drosophila, only one neurexin (dnrx) gene has been found, and this gene is critical for the formation of synapse cytoarchitecture, synapse development, and function (Li et al., 2007; Zeng et al., 2007). However, the spatial and temporal distribution of Drosophila neurexin

*Corresponding author (email: sunmingkuan@gmail.com)
(DNRX) is still not well characterized.

Previous studies showed that the dnrx mRNA was enriched in the brain and in the ventral nerve cord by in situ hybridization (Chen et al., 2010; Li et al., 2007). However, it is difficult to detect whether $d n r x$ expresses in muscle cells using RNA in situ hybridization (Li et al., 2007; Zeng et al., 2007). The subcellular localization of DNRX has been investigated in embryos, larval brains using regular antibody staining, and DNRX was concentrated in the neuropil regions of the brain and the ventral nerve cord in embryos and larval brains (Li et al., 2007; Sun et al., 2009; Zeng et al., 2007). In the adult brain, DNRX was localized in several brain lobes (Zeng et al., 2007). DNRX is a synaptic protein and is transported along axons to synaptic terminals after it has been expressed in the cell body. Therefore it cannot be precisely depicted using regular antibody staining 
from which cells DNRX expression in neuropil transport. In addition, DNRX expression is undetectable in muscle cells using regular antibody staining because of a low level of DNRX protein at the NMJs and/or a low affinity of DNRX antibodies.

The GAL4-UAS (GAL4-Upstream activating sequence) ectopic expression system is widely used in Drosophila melanogaster for gene expression and functional analysis. The GAL4-UAS system for gene expression analysis allows for the overcoming of some of the limitations of in situ hybridization and antibody staining. Therefore, we generated the DNRX-Gal4 line, which fuses the core promoter sequence of the dnrx gene with Gal4, to confirm DNRX expression in NMJs and to analyze the cell specific expression pattern of DNRX during the fly life cycle.

\section{RESULTS}

\section{Expression pattern of DNRX-Gal4 was similar to en- dogenous DNRX}

We established four independent transgenic lines bearing the DNRX-Gal4 construct, which spanned the region from -357 to +1711 (Figure 1A) (Sun et al., 2009). To compare DNRX-Gal4 expression with endogenous DNRX, DNRXGal4 transgenic flies were crossed with the UAS-mCD8GFP on wild type background and with the DNRX overexpression allele on a $d n r x$ mutant background. The third larvae brain was co-stained with anti-CD8 and DNRX antibody or anti-Dnlg2 (Sun et al., 2011) and DNRX antibody. The axon, synaptic neuropil and the cell body of motor neurons were labeled by anti-CD8 antibody. Endogenous DNRX was expressed in synaptic neuropil where DNRX signal was colocalized with CD8-GFP driven by DNRXGal4 (Figure 1B). Meanwhile our results indicated that exogenous DNRX driven by DNRX-Gal4 was correctly distributed in the neuropil of the larval brain, similar to endogenous DNRX (Figure 1C and D). Taken together, these results indicated that DNRX-Gal4 drove gene expression in a pattern similar to the endogenous DNRX expression pattern in the larval CNS.

\section{DNRX was expressed in motor neurons during all stages of development}

To characterize the expression pattern of DNRX throughout the entire life cycle, DNRX-Gal4 transgenic flies were crossed with the UAS-mCD8-GFP reporter line. The reporter expression pattern was tested using immunostaining with a GFP antibody. To determine the initiation of DNRX expression, we stained the embryos (genotype: DNRXGal4/+; UAS-mCD8-GFP/+) at various stages with the anti-Elav antibody to mark differentiated neurons. The results demonstrate that the GFP signal can be detected in a subset of neurons in the ventral nerve cord as early as stage 15 , a point at which motor neuron differentiation has already occurred (Figure 2A). More GFP-positive neurons occurred at very late stages (beyond stage 17, Figure $2 \mathrm{~B}$ ).

In the third instar larval stage, the ventral nerve cord (thick arrow) and motor nerves (arrow) can be seen through the translucent cuticle in a composite image of the third instar larva (genotype: DNRX-Gal4/+; UAS-mCD8$\mathrm{GFP} /+$ ). Individual nerve branches and neuromuscular junctions (NMJs) can be easily identified under higher magnification in the third instar larval stage (Figure 3A and B). Our results indicate that the GFP reporter was expressed in the leg disc (arrow in Figure 3C). In the adult leg, we were able to image the innervation pattern of the muscles through the cuticle. The GFP reporter was expressed in the sensory neurons of each leg (thick arrow in Figure 3F). The sensory neurons at the anterior margin of the wing were also mCD8-GFP positive (thick arrow in Figure 3G). In addition, we found that the GFP reporter was expressed in a few non-neuronal cells in the mid-gut epithelium (Figure 3D) and in the salivary glands (Figure 3E). In summary, the DNRX-Gal4 line expresses Gal4 most prominently in neurons that innervate muscles in embryonic and adult stages. These results suggested that DNRX was expressed in motor neurons throughout the entire life cycle.

The larval brain forms during the first $24 \mathrm{~h}$ of embryogenesis. Subsequently, Drosophila has three larval stages (L1, L2 and L3) before its pupation and then it reaches adulthood. The larval brain already contains the anlagen for the following adult brain structures: the mushroom bodies (MBs), optic ganglia and antennal lobe. At the third larval stage, the MBs appear as paired neuropiles with a structure mostly conserved in the adult. The Kenyon cells (Kc) extend two types of processes. One type forms a dendritic calyx $(\mathrm{Ca})$; the other forms a fasciculated axonal tract (the peduncle), which extends ventrally toward the anterior surface of the brain, where it separates into two main branches: the dorsal lobe (Dl) and the medial lobe (Ml).

DNRX-Gal4 expression (monitored through mCD8-GFP or GFP-lacZ.nls expression) was compared with the staining of Elav in the larval brain and ventral nerve cord. Our results demonstrate that mCD8-GFP was highly expressed in the ventral nerve cord, brain lobes and motor neuron axon bundles (Figure 4A). In addition, enlargement of the brain demonstrated that the mCD8-GFP signal was expressed in the calyx and lobes of the MBs (Figure 4A'), which suggests that DNRX-Gal4 was expressed in the MBs. Furthermore, we analyzed the expression of GFP-lacZ.nls driven by DNRX-Gal4 in the brain during the third larval instar. Our results show that the GFP-lacZ.nls signal was expressed in MBs'neurons (KCs) (Figure 4B). These results indicate that DNRX was highly expressed in MB's neurons (KC neurons).

We next wanted to determine whether DNRX was expressed in all neurons or only in a subset of neurons. To 
address this question, we performed double staining using an anti-GFP antibody and an anti-Elav antibody (a panneuronal marker) in a DNRX-Gal4/+; UAS-GFPlacZ.nls/+larval brain. Our results demonstrated that the expression of DNRX-Gal4 was highly selective and marked only a subset of cells from a large field of neurons identified using the pan-neuronal marker Elav in the ventral ganglion of third instar larvae (Figure 4C). In summary, DNRX was

A dnrx gene span
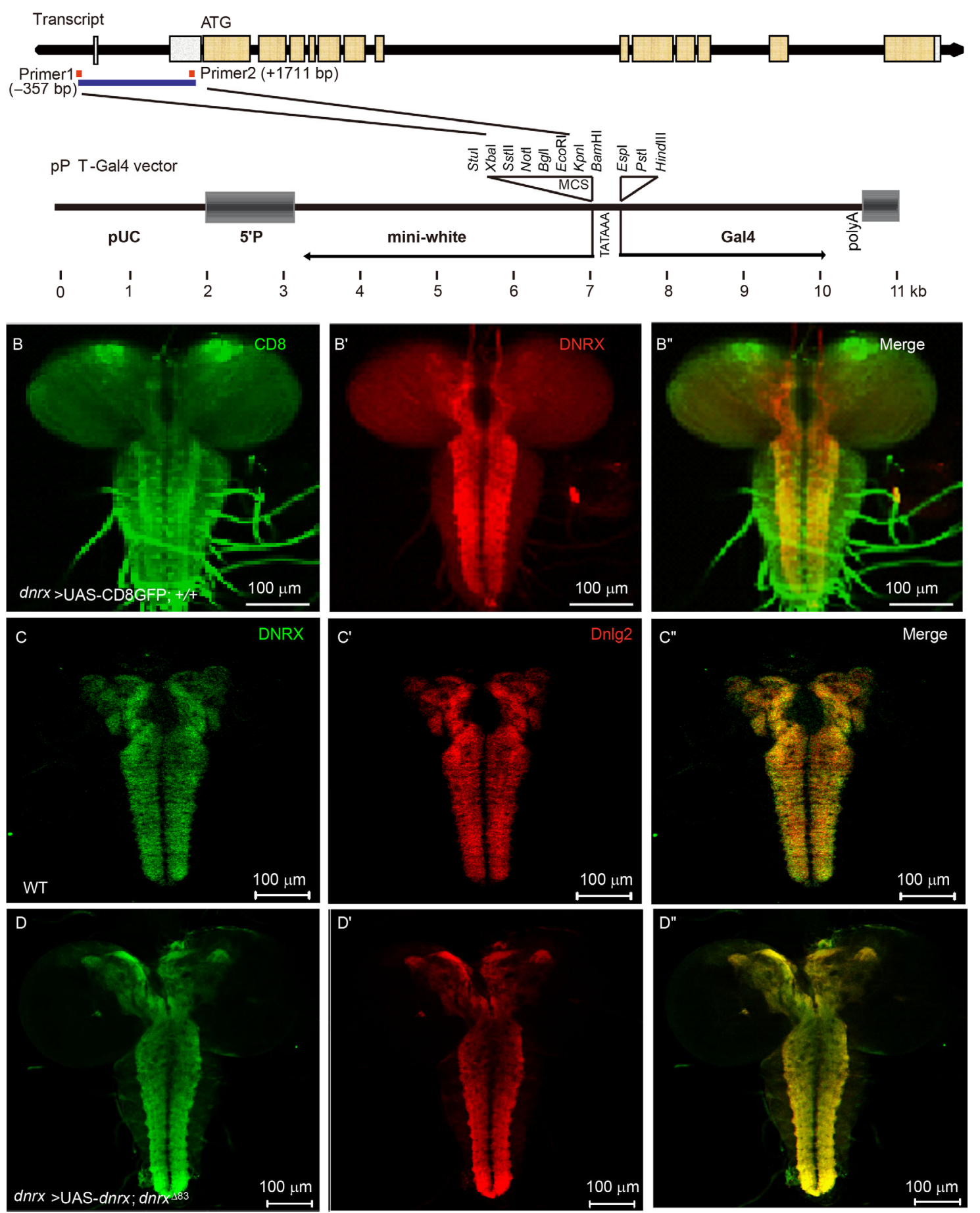

Figure 1 Fragments of the dnrx enhancer and map of the pPT-GAL vector. A, The genomic region $357 \mathrm{bp}$ upstream of the transcription start site to the ATG was fused to the pPT-GAL vector. The resultant construct (bottom), termed DNRX-Gal4, was used for P-element mediated transformation, and independent transgenic lines were established. B, The brain disc of DNRX-Gal4/UAS-CD8-GFP was stained with anti-CD8 (green) and anti-DNRX antibody (red). C and D, In third instar larvae brain, DNRX-Gal4 had the same expression pattern as endogenous DNRX. 

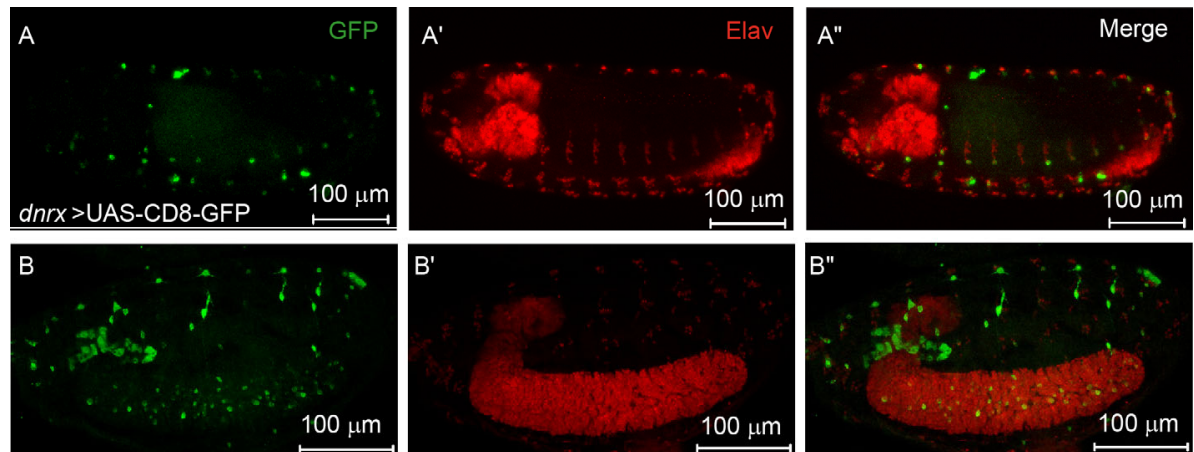

Figure 2 (color online) Expression pattern of DNRX-Gal4 in the embryo. A, Lateral view of a stage 12 embryo; GFP expression is detectable in early-born motor neurons. B, Lateral view of a stage 16 embryo showing DNRX-Gal4 expression in the brain (br) and ventral nerve cord (VNC).
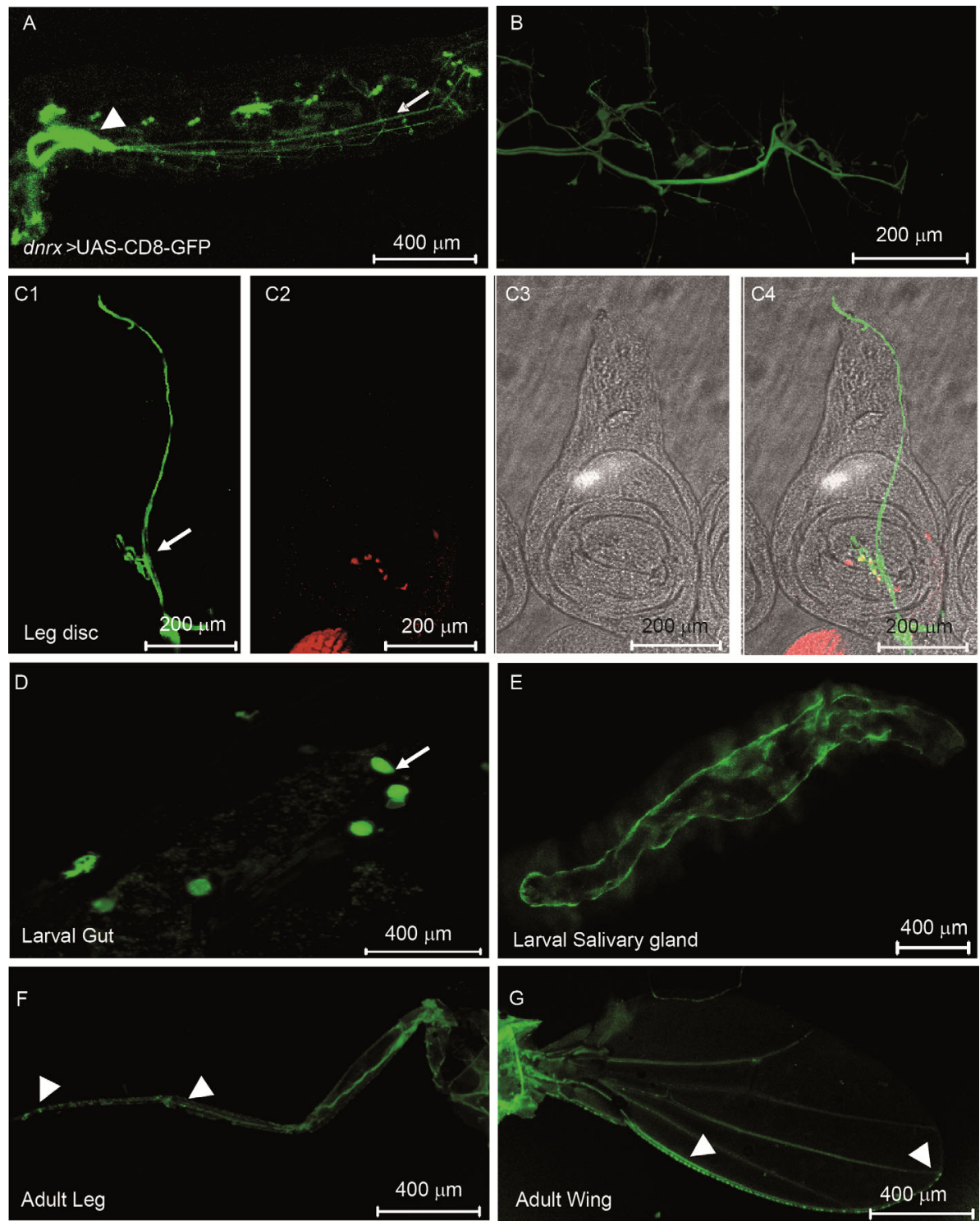

Figure 3 (color online) Confocal micrographs of the DNRX-Gal4 expression pattern throughout the life cycle. A and B, In a composite image of a third instar larva, the ventral nerve cord (thick arrow head) and the motor nerves (arrow) can be seen through the translucent cuticle. C, DNRX-Gal4 was expressed in the leg imaginal discs of Drosophila third instar larvae. D, Relatively small, non-neuronal cells (arrow) in the epithelium of the midgut of a third instar larva. E, DNRX-Gal4 was also detected in the salivary glands. F, The innervation pattern of the adult leg musculature can be observed through the cuticle. A few sensory neurons in the tarsi (thick arrow head) can also be seen. G, In adult wings, chemosensory cells at the anterior margin project via the wing nerve to the brain. 

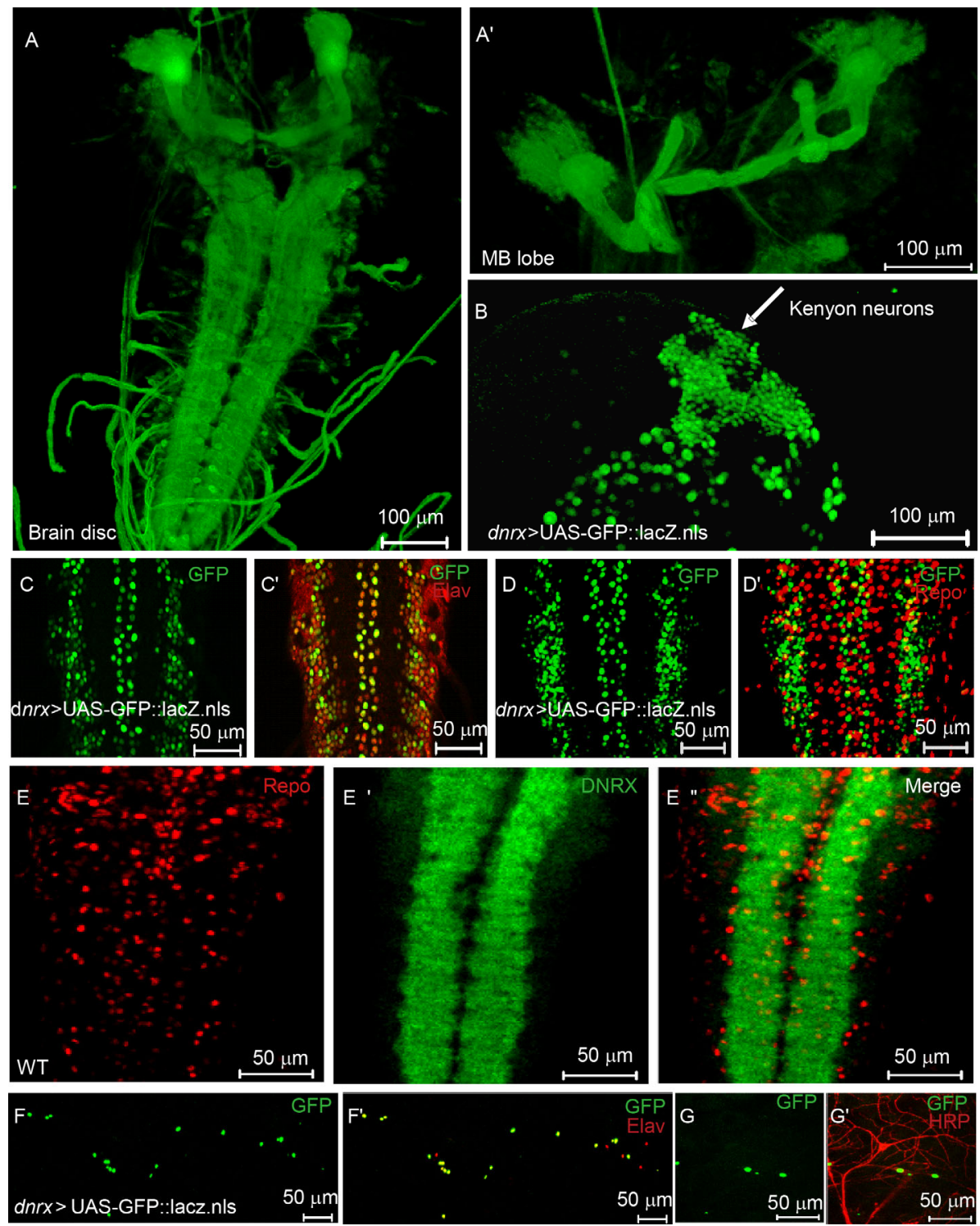

Figure 4 (color online) DNRX-Gal4 is predominantly restricted to a subset of neurons, not glia cells. A and B, Expression of DNRX-Gal4 in the larvae brain (MBs and neuropil). C, Images of the ventral nerve cord double labeled to reveal DNRX-Gal4-driven nuclear GFP (C) and Elav-expressing neurons (C') showing that DNRX-Gal4 is expressed in some Elav-positive neurons. D, Double-labeled images with DNRX-Gal4-driven nuclear GFP ( $\left.\mathrm{D}^{\prime}\right)$ and an anti-Repo antibody revealed that DNRX-Gal4 is not expressed in glial cells. E, Confocal images of larval brain VNC costained with anti-Repo and anti-DRNX showing completely different expression pattern between DNRX and Repo. F and G, DNRX-Gal4 is also expressed in some sensory neurons.

expressed in some, but not all, neurons.

To analyze whether DNRX was expressed in glial cells, we performed double staining with an anti-GFP antibody and an anti-Repo antibody in the larval brain (Genotype: DNRX-Gal4/+; UAS-GFP-lacZ.nls/+). Our staining results demonstrated that the GFP reporter and Repo signal were not colocalized (Figure 4D) in the ventral nerve cord. Furthermore, we performed double staining with an anti-DNRX antibody and an anti-Repo antibody in the wild type third instar larval brain. Our result showed that the expression pattern of endogenous DNRX was different from that of Repo (Figure 4E). These results suggest that DNRX is not expressed in glial cells.

To analyze whether DNRX was expressed in peripheral sensory neurons, we crossed DNRX-Gal4 transgenic flies to UAS-GFP-lacZ.nls flies and analyzed the GFP expression pattern. The peripheral sensory neurons were labeled by GFP-lacZ.nls (Figure 4F). This result indicates that DNRX is also expressed in sensory neurons. Additionally, we found that some Elav-positive sensory neurons lacked GFP expression, which also suggests that DNRX is not expressed in all sensory neurons. Furthermore, we co-stained the dendrites and axons of sensory neurons with an anti-GFP antibody and an anti-HRP antibody. These results confirmed that DNRX-Gal4 was expressed in some, but not all of the sensory neurons (Figure 4G). These results also suggest that DNRX might be expressed and play a role in peripheral sensory neurons. 
DNRX was expressed in pre- and post-synapses in third instar larvae neuromuscular junctions

Endogenous DNRX may be present both pre- and postsynaptically in the embryo. Thus, we next asked whether DNRX was expressed in both pre- and post-synapses in the third instar larval NMJs.

To address this question, we analyzed the expression of DNRX using the DNRX-Gal4/UAS-GFP-lacZ.nls reporter system followed by immuno-staining with an anti-GFP antibody. The muscle cell nuclei were stained with DAPI (4',6-diamidino-2-phenylindole). The staining results demonstrated that GFP-lacZ.nls was highly expressed in the nucleus of third instar larval muscle cells and that the expression was driven by the DNRX-Gal4 promoter (Figure 5B). There was no GFP expression in the nuclei of nonDNRX-Gal4-driven third instar larval muscle cells (Figure 5A). These results demonstrate that DNRX is expressed in the muscle, which indicates that DNRX could be expressed in post-synaptic areas in the third instar larval NMJs.

Furthermore, we investigated the expression of DNRX in the NMJ using immuno-electron microscopy (IEM). We first analyzed the expression of endogenous DNRX using an anti-DNRX antibody in 24 NMJs from three different experiments. Black immunogold particles indicated that DNRX was present in the presynaptic nerve terminals and the postsynaptic muscle cells. As shown in Figure 5C, about $70 \%$ black immunogold particles were found in the postsynaptic muscle (blue arrow) and 30\% black immunogold particles were localized in the presynaptic terminals (red arrow).

The expression level of endogenous DNRX at NMJs is very low [6,8]; only a few immunogold particles can be detected in wildtype NMJs. Therefore we expressed Drosophila exogenous neurexin using a DNRX-Gal4 driver, and then, we analyzed the expression of DNRX using IEM. Our IEM results demonstrated that exogenous DNRX was expressed in both pre- (Figure 5D, red arrow) and postsynaptic regions (Figure 5D, blue arrow) in third instar larvae NMJs. Together, our results indicate that DNRX is expressed in, not only pre-synaptic neurons, but also post-synaptic muscle cells. Thus, DNRX-Gal4 would be a very useful genetic tool for co-expression in both neurons and muscle to study the development of the NMJs.

\section{Whole-brain distribution of DNRX expressing cells in adults}

To map the DNRX-expressing cells in the adult brain, the DNRX-Gal4 line was crossed with the UAS-GFP-lacZ.nls reporter line, a line which expresses nuclear localized GFP in GAL4-expressing cells. As shown in Figure 6A and C, DNRX-Gal4-driven nuclear-localized GFP expression was observed in a wide variety of cell types throughout the brain, including MBs (Kenyon neurons), antennal lobe neurons, optic
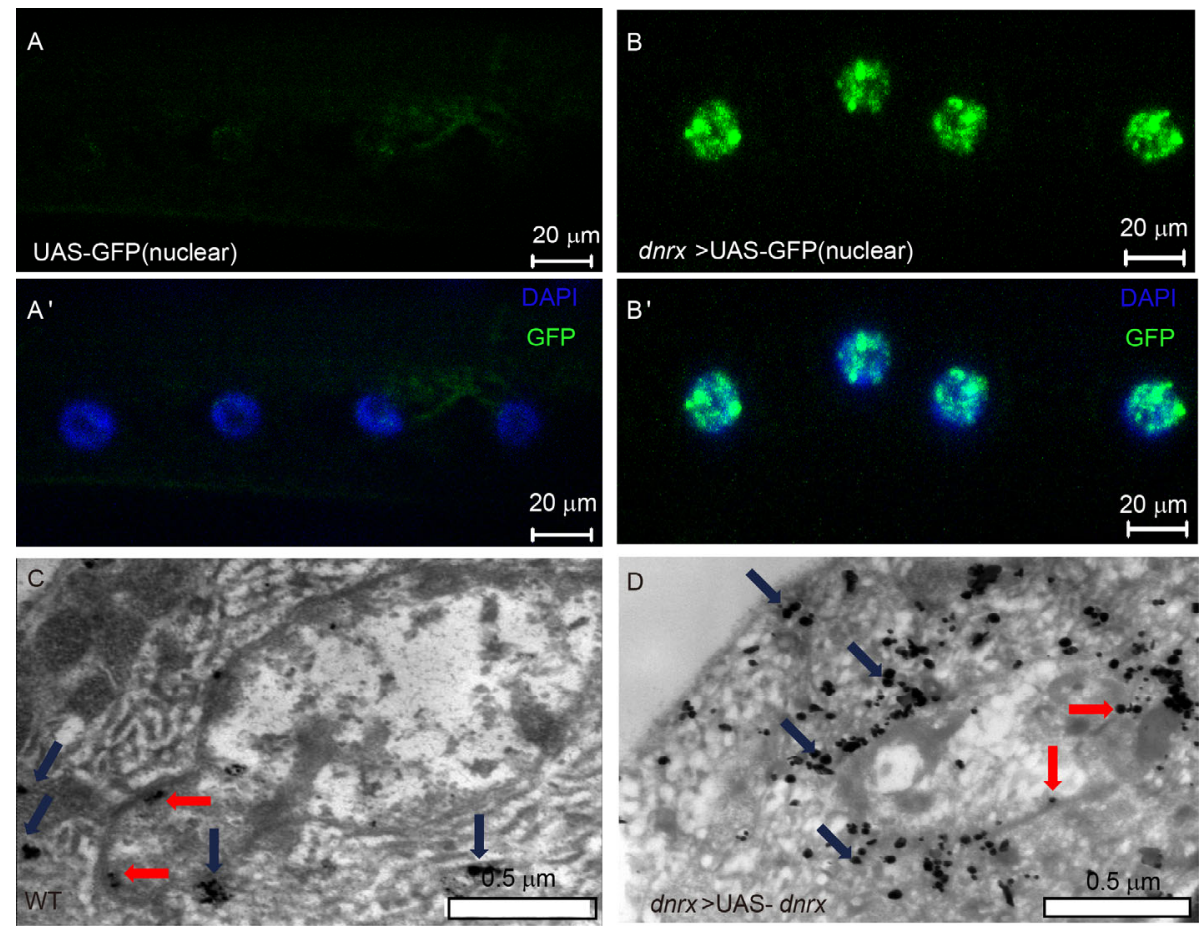

Figure 5 DNRX-Gal4 is expressed in pre- and post-synaptic areas in the Drosophila neuromuscular junctions. A and B, The expression of GFP driven by DNRX-Gal4 in the muscle cell nuclei, which indicates that DNRX-Gal4 is expressed in both pre- and post-synaptic areas in the NMJs. C, Representative immune-electron micrograph from WT NMJs showing endogenous DNRX immunogold particles in both presynaptic terminals (red arrow) and in postsynaptic muscle (blue arrow). D, Representative immune-electron micrograph from $d n r x>\mathrm{UAS}-d n r x$ NMJs revealed that exogenous DNRX driven by DNRX-Gal4 is localized to the pre- (red arrow) and postsynaptic areas (blue arrow). 

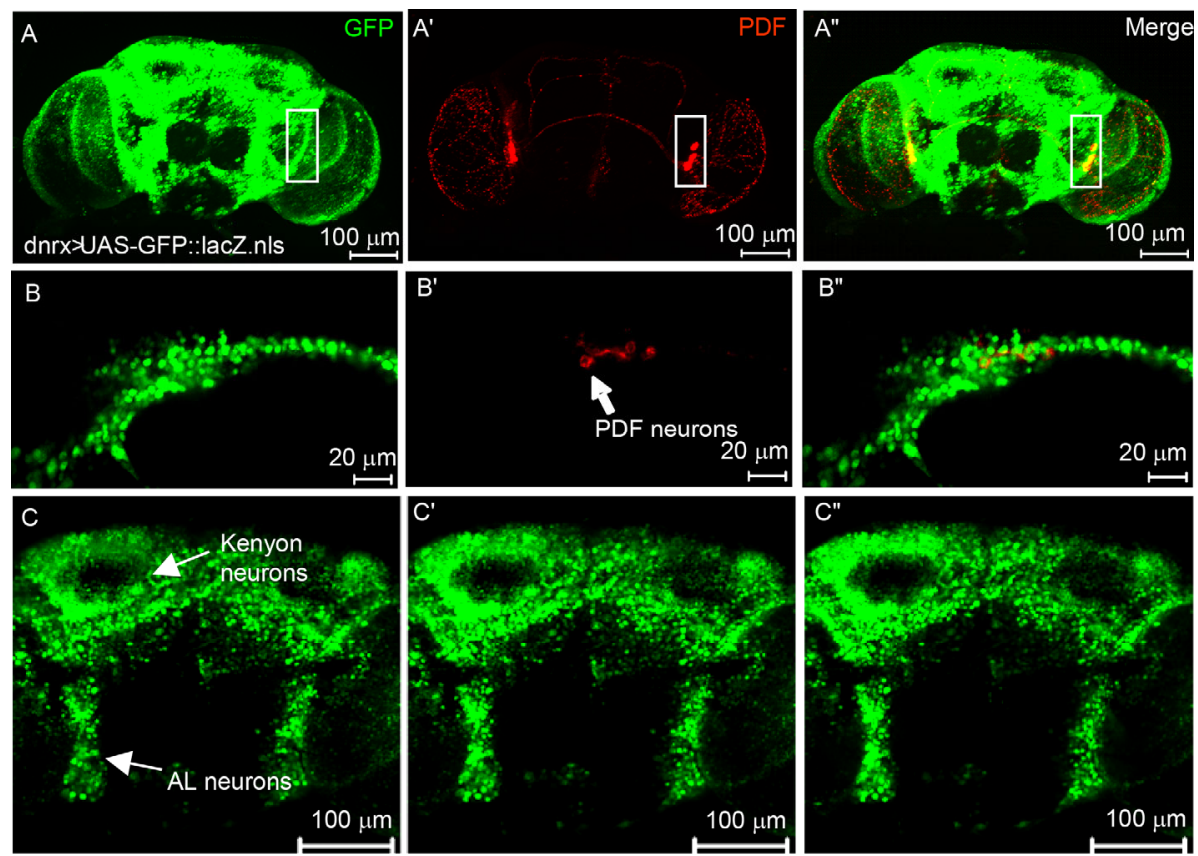

Figure 6 (color online) Whole-brain distribution of DNRX-Gal4 expressing cells in adults. A and B, Expression of DNRX-GAL4 was observed in PDF-positive neurons identified by double staining with an anti-PDF antibody. PDF-positive neurons are indicated by arrows (B). C, Expression of DNRX-Gal4 in Kenyon neurons and antennal lobe neurons.

ganglion neurons and the sub-esophageal ganglion (SOG).

The antennal lobe is the primary olfactory center within the Drosophila brain and represents the anatomical and functional equivalent of the vertebrate olfactory bulb. DNRX expression in antennal lobe suggests that DNRX might play a role in the development of the antennal lobe. To visualize the distribution of endogenous DNRX in the brain, we performed antibody staining of the entire Drosophila head. Our results demonstrated that DNRX was predominantly localized in the brain, including $\mathrm{MBs}$, the antennal lobe (Figure 7A), the medulla, and the lobula complex (Figure 7B).

In Drosophila and other animals, the circadian clock is synchronized to the environmental light cycle and supervises the daily activity rhythms in physiology and behavior. Drosophila circadian rhythms are controlled by a neural circuit containing approximately 150 clock neurons. We found that DNRX was expressed in PDF-positive clock neurons (Figure 6A and B). This result suggests that DNRX might play a role in Drosophila rhythms.

\section{DNRX expression in MBs was required for olfactory learning}

Numerous studies have indicated that the Drosophila MBs are critical for olfactory associative learning and memory (Akalal et al., 2006; McGuire et al., 2001). The immunostaining results with the DNRX antibody and reporter expression pattern by DNRX-GAL4 demonstrate that DNRX was strongly expressed in MBs within the Drosoph- ila adult brain (Figure 7A and C). Whether DNRX is involved in learning and memory during the adult stage is unknown. Here, we used the T-maze to test the learning and memory ability of Drosophila adults carrying a $\mathrm{P}$ element or null mutation of the dnrx gene. The dnrx mutants exhibited normal responses to odors and electric shocks (Figure 7D and E). However, the $d n r x$ mutants with a $\mathrm{P}$ element insert had a low learning and memory index compared with the wild type adults (WT, $L I=66.4 \pm 1.07, n=8 v s$. $d n r x^{\mathrm{d} 08766}$, $L I=49.7 \pm 1.48, n=8, P<0.05$; Figure $7 \mathrm{~F})$. The homozygous dnrx null mutants, $d n r x^{\Delta 83 / \Delta 83}$, demonstrated a lower learning and memory ability $\left(d n r x^{\Delta 83 / \Delta 83}, L I=21.0 \pm 0.82, n=8 v s\right.$. WT, $P<0.01$; Figure $7 F$ ). These results suggest that the learning and memory ability of Drosophila adults may depend on DNRX expression.

To address this question of whether DNRX expression in MBs play an important role in Drosophila learning and memory, we performed MBs special Gal4 (Ok107-Gal4) rescue experiment. Here, we also first assessed the responses of the different genotypes used in our MBs rescue experiment for normal odor and shock avoidance; no significant differences were observed for odor and shock avoidance between these flies (Figure 7D and E). $d n r x^{\Delta 83 / \Delta 83}$ flies bearing one OK107-Gal4 (genotype: $d n r x^{\Delta 83 / \Delta 83}$; OK107Gal4/+ and $L I=21.2 \pm 0.42, n=8$ ) or one UAS- $d n r x$ (genotype: UAS- $d n r x /+; d n r x^{\Delta 83 / \Delta 83}$ and $\left.L I=19.3 \pm 0.36, n=8\right)$ or one DNRX-Gal4 (genotype: DNRX-Gal4/+; $d n r x^{\Delta 83 / \Delta 83}$ and $L I=20.9 \pm 0.92, n=8$ ) displayed poor learning and memory. This is the same as the $d n r x$ null mutant (Figure 7F). MBs special rescue flies (genotype: UAS-dnrx/+; $d n r x^{\Delta 83 / \Delta 83}$; 

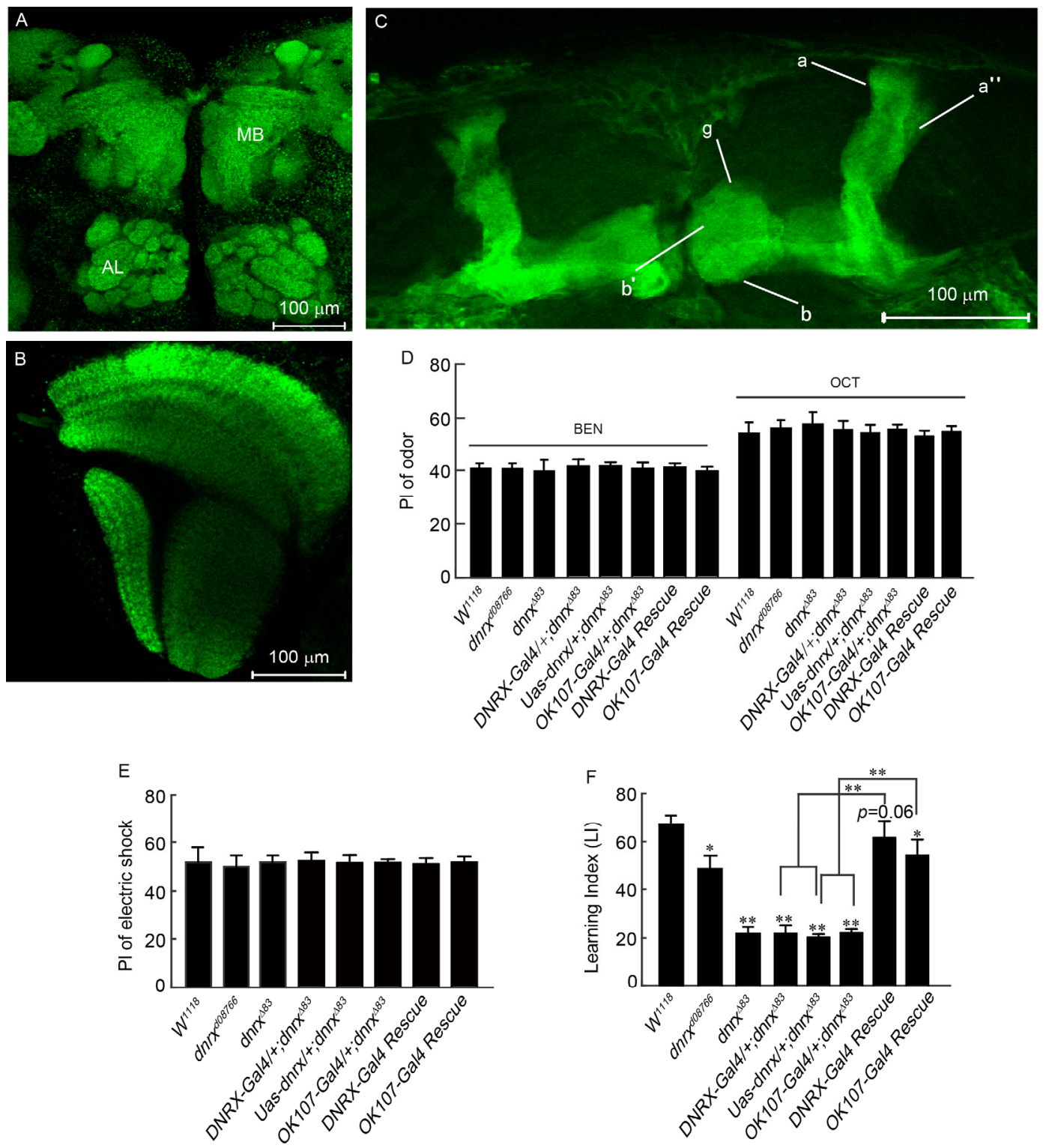

Figure 7 (color online) MBs expression of DNRX-Gal4 agreed with the DNRX mapping of the dose-dependent memory circuits in olfactory conditioning. A and C, Endogenous DNRX and DNRX-Gal4 are highly expressed in adult MBs. B, DNRX is expressed in Drosophila optic lobes. D, E and F, Flies that were two to three days old were assayed immediately after the training session with the classical conditioning procedure developed by Tully and Quinn (1985). Compared with wild-type Drosophila $w^{1118}$, the $d n r x$ P element insertion mutants had lower $L I$ values, and the null mutant $d n r x^{\Delta 83}$ had more severe learning defects (D, E and F). The Learning and memory defects of $d n r x$ null mutants can be partially rescued by DNRX expression in the MBs and restored to wild type level by DNRX expression driven by DNRX-Gal4. These results support the observed expression of the reporter gene driven by DNRX-Gal4.

OK107-Ga4/+ and $L I=52.3 \pm 1.92, n=8)$ performed significantly better than control flies (genotype: $d n r x^{\Delta 83 / \Delta 83}$; OK107-Gal4/+ and UAS- $\left.d n r x /+; d n r x^{\Delta 83 / \Delta 83}\right)$. These results showed that the learning and memory defects of $d n r x$ null mutants can be partially rescued by DNRX expression in MBs using OK107-Gal4. Our results suggest that DNRX expression in MBs is required for Drosophila learning and memory.

If the DNRX expression driven by DNRX-GAL4 is consistent with the endogenous DNRX expression pattern, it should be able to rescue the learning and memory defects of dnrx null mutants. To test this hypothesis, we performed the rescue experiment using DNRX-Gal4 as a driver. Our results showed that DNRX expression driven by DNRX-Gal4 (genotype: DNRX-Gal4/UAS-dnrx; $\quad d n r x^{\Delta 83 / \Delta 83}$ and $L I=$ $59.4 \pm 1.9, n=8$ ) was sufficient in restoring the learning and memory of dnrx null mutant flies to wild type level (Figure $7 \mathrm{~F})$.

\section{DISCUSSION}

We have reported the DNRX-Gal4 transgenic lines under the control of the $d n r x$ gene regulatory region in our previ- 
ous publication. Here, we characterized the expression of the DNRX-Gal4 transgenic line. Although several papers have reported the endogenous DNRX expression pattern using DNRX specific antibodies (Chen et al., 2010; Li et al., 2007; Sun et al., 2009; Tian et al., 2013; Zeng et al., 2007), the DNRX expression in various tissues during fly development has not been described. We utilized the Gal4/UAS system to examine the temporal and spatial expression of DNRX during the fly life cycle.

We found that DNRX expression driven by DNRX-Gal4 was compatible with endogenous DNRX expression in the third instar larvae brain. Thus, we analyzed the temporal and spatial expression of DNRX using a reporter gene driven by DNRX-Gal4 in the embryonic and larval stages and in non-neuronal and neuronal tissues in the adult stage. We found that DNRX-Gal4 was initially expressed in a subset of neurons at stage 15 of Drosophila embryonic development.

Neurexins are also localized in the post-synaptic areas. Neurexins expressed in the post-synaptic areas may control synaptogenesis by silencing the function of post-synaptic Nlgs [18]. Chen et al. (Chen et al., 2010) found that DNRX was expressed in embryo post-synaptic body wall muscles using RNA in situ hybridization. Functionally, the post-synaptic RNAi knockdown of neurexin changed the abundance of the post-synaptic glutamate receptor protein. This finding provided new insights into the role of neurexin in synapse development. Our results also indicated that DNRX was expressed in the muscle cells of the third instar larvae using the DNRX-Gal4/UAS-reporter system (Figure $5 \mathrm{~A}$ and $\mathrm{B}$ ). Furthermore, our immune-electron microscopy results with an anti-DNRX specific antibody indicated that both endogenous DNRX and exogenous DNRX driven by DNRX-Gal4 were localized to the post-synaptic areas of the Drosophila larval NMJs (Figure 5C and D). Therefore, our results provide new evidence about the post-synaptic function of neurexins. Additionally, we prepared a very useful Gal4 tool for co-expression in both neurons and muscle.

Our DNRX expression pattern analyses indicate that DNRX is expressed in a subset of neurons, but not in glia, throughout the life cycle (Figure 1-4). Specifically, DNRX was highly expressed in the MBs of both the larval and adult brain. DNRX may be involved in MBs plasticity and function during the larval stage, which is consistent with a previous report (Zeng et al., 2007). Furthermore, our results indicated that DNRX function was a dose-dependent regulator in adult learning and memory.

\section{MATERIAL AND METHODS}

\section{Fly stocks}

Flies were maintained at $25^{\circ} \mathrm{C}$ on a standard medium with $60 \%-80 \%$ relative humidity. $W^{1118}$ flies were used as a wild-type strain. The fly stocks 6451 (UAS-GFP-lacz.nls) and 5137 (UAS-mCD8-GFP) were obtained from the Bloomington Stock Center at Indiana University (Bloomington, USA).

\section{Plasmid construction and transgenic flies}

DNRX-Gal4 lines have been described in our previous publication (Sun et al., 2009). Analyses of $d n r x$ promoter region were performed using the promoter prediction program (NNPP version 2.2, Berkeley Drosophila Genome Project, http://www.fruitfly.org/index.html) (Huang et al., 2014; Reese, 2001). The genomic fragment including the $d n r x$ core promoter was amplified and cloned into the pPTGAL (Sharma et al., 2002) [12] vector.

\section{Reporter signal detection and antibody staining}

To detect DNRX-Gal4 expression in the third instar larval stage, transgenic flies were crossed with the UAS-mCD8-GFP and UAS-GFP-lacz.nls. Confocal analysis was performed on a Zeiss confocal station and imaged with the LSM510 software (Zeiss, Germany).

The embryos were dechorionated for 4 min with $2 \%$ sodiumhypochlorite (bleach), fixed with fresh $3.7 \%$ formaldehyde (in phosphate buffered solution (PBS))/heptane for $20 \mathrm{~min}$ and devitellinized by shaking in methanol/heptane. The embryos were rehydrated with $0.1 \%$ PBT (PBS containing $0.1 \%$ Triton X-100), blocked with $0.1 \%$ PBT $/ 5 \%$ bovine serum albumin (BSA) and incubated overnight with the primary antibody in $0.1 \%$ PBT (Zeng et al., 2007).

Wandering third instar larval brains and adult brains were dissected in $1 \times \mathrm{PBS}$ and fixed in $4 \%$ paraformaldehyde for $40 \mathrm{~min}$. After $3 \times 5 \mathrm{~min}$ washes, the preparations were blocked with $5 \%$ BSA in $0.1 \mathrm{~mol} \mathrm{~L}^{-1}$ phosphate buffer $(\mathrm{pH}$ 7.2) containing $0.3 \%$ TritonX-100 for $1 \mathrm{~h}$. Next, the primary antibodies were added and incubated overnight at $4^{\circ} \mathrm{C}$.

The primary antibodies were diluted as follows: mouse anti-Elav (DSHB, USA), 1:50; mouse anti-Repo (DSHB, USA), 1:50; rabbit anti-GFP, 1:2000 (Torrey Pines Biolabs, USA); rabbit anti-DNRX antibody, 1:200; and mouse anti-DNRX mAb A4, 1:50 (Sun et al., 2011).

\section{Immunogold electron microscopy}

Larval NMJs were dissected in HL3.1 buffer $\left(128 \mathrm{mmol} \mathrm{L}^{-1}\right.$

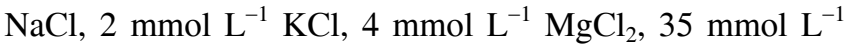

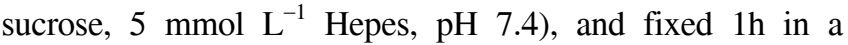
mixture of $2 \%$ glutaraldehyde and $2 \%$ formaldehyde in $0.1 \mathrm{~mol} \mathrm{~L}^{-1}$ sodium cacodylate buffer ( $\mathrm{pH} 7.4$, wash buffer). Specimens were washed four times with wash buffer and blocked $1 \mathrm{~h}$ at $4{ }^{\circ} \mathrm{C}$ with block buffer $(10 \%$ New goat serum $+1 \%$ BSA $+0.1 \%$ saponin). Specimens were incubated overnight at $4^{\circ} \mathrm{C}$ in DNRX antibody, washed with $0.1 \%$ saponin 
buffer, and incubated for $1 \mathrm{~h}$ at room temperature in secondary antibody conjugated to $0.8 \mathrm{~nm}$ gold particles $(1 / 50$ dilution, Nanoprobes, USA). After being thoroughly washed 3 times with $\mathrm{ddH}_{2} \mathrm{O}$, silver enhancement was performed (HQ Silver enhancement kit, Nanoprobes). Specimens were transferred to $1 \%$ osmium tetroxide in $0.1 \mathrm{~mol} \mathrm{~L}^{-1}$ sodium cacodylate ( $\mathrm{pH}$ 7.4) for $2 \mathrm{~h}$, washed three times with $\mathrm{ddH}_{2} \mathrm{O}$, and dehydrated in an ethanol series, and embedded into Lowicry1 K4M. Ultra-thin sections (90 nm thick) were cut with Leica UC6 (Germany) using a diamond knife. Grids were post-stained 2 min with $2 \%$ saturated uranyl acetate in $50 \%$ ethanol and 2 min with $1 \%$ lead citrate ( $\mathrm{pH} \mathrm{12)}$, examined with H-7650 electron microscope (Hetachi, Japan) and recorded with a Ganton 830 digital CCD.

\section{Learning experiments}

The learning experiments were performed as described by Tully and Quinn (Tully and Quinn, 1985). Briefly, three to five days old flies were sequentially exposed to two odors for $60 \mathrm{~s}$ each. The first odor was paired with an electric shock $(60 \mathrm{~V}, 12 \times 1.5 \mathrm{~s}$ pulses), and the second odor was not paired. The associative learning was tested within 3 min of training completion. During the testing, flies were exposed to both odors simultaneously in a T-maze. All training and testing were performed in a climate-controlled room with $75 \%$ humidity at $25^{\circ} \mathrm{C}$ under a dim red light. The learning index was calculated as the percentage of flies that correctly avoided the odor paired with an electric shock minus the percentage that incorrectly avoided the second odor. The paired and unpaired odors were swapped in each half of the experiment. The performance index (PI) was calculated as the average of the two reciprocal learning index values. Sensorimotor responses were tested in untrained flies of the same age. Flies were placed in a T-maze and given a choice between an odor and clean air or between two electric grids (with only one of the grids connected to the stimulator). The avoidance values represent the percentage of flies that avoided the odor or electric shock minus the percentage of flies that did not. The genotypes of the experimental flies were coded before the experiments such that the experiments were performed blind to avoid any subconscious bias.

Compliance and ethics The author(s) declare that they have no conflict of interest.

Acknowledgements We thank the Bloomington Stock Center for the fly stocks. This work was supported by the National Natural Science Foundation of China (31171041, 31000486) and the National Basic Research Program of China (2012CB517903).

Akalal, D.B., Wilson, C.F., Zong, L., Tanaka, N.K., Ito, K., and Davis, R.L. (2006). Roles for Drosophila mushroom body neurons in olfactory learning and memory. Learn Mem 13, 659-668.

Chen, K., Gracheva, E.O., Yu, S.C., Sheng, Q., Richmond, J., and Featherstone, D.E. (2010). Neurexin in embryonic Drosophila neuromuscular junctions. PloS One 5, e11115.

Dean, C., Scholl, F.G., Choih, J., DeMaria, S., Berger, J., Isacoff, E., and Scheiffele, P. (2003). Neurexin mediates the assembly of presynaptic terminals. Nature Neurosci 6, 708-716.

Graf, E.R., Zhang, X., Jin, S.X., Linhoff, M.W., and Craig, A.M. (2004). Neurexins induce differentiation of GABA and glutamate postsynaptic specializations via neuroligins. Cell 119, 1013-1026.

Huang, W.L., Tung, C.W., Liaw, C., Huang, H.L., and Ho, S.Y. (2014). Rule-based knowledge acquisition method for promoter prediction in human and Drosophila species. Sci World J 2014, 327306.

Li, J., Ashley, J., Budnik, V., and Bhat, M.A. (2007). Crucial role of Drosophila neurexin in proper active zone apposition to postsynaptic densities, synaptic growth, and synaptic transmission. Neuron 55, 741-755.

McGuire, S.E., Le, P.T., and Davis, R.L. (2001). The role of Drosophila mushroom body signaling in olfactory memory. Science 293, 1330-1333.

Missler, M., Fernandez-Chacon, R., and Sudhof, T.C. (1998). The making of neurexins. J Neurochem 71, 1339-1347.

Missler, M., Zhang, W., Rohlmann, A., Kattenstroth, G., Hammer, R.E., Gottmann, K., and Sudhof, T.C. (2003). Alpha-neurexins couple $\mathrm{Ca}^{2+}$ channels to synaptic vesicle exocytosis. Nature 423, 939-948.

Reese, M.G. (2001). Application of a time-delay neural network to promoter annotation in the Drosophila melanogaster genome. Com Chem 26, 51-56.

Sharma, Y., Cheung, U., Larsen, E.W., and Eberl, D.F. (2002). PPTGAL, a convenient Gal4 P-element vector for testing expression of enhancer fragments in drosophila. Genesis 34, 115-118.

Sun, M., Liu, L., Zeng, X., Xu, M., Liu, L., Fang, M., and Xie, W. (2009). Genetic interaction between Neurexin and CAKI/CMG is important for synaptic function in Drosophila neuromuscular junction. Neurosci Res 64, 362-371.

Sun, M., Xing, G., Yuan, L., Gan, G., Knight, D., With, S.I., He, C., Han, J., Zeng, X., Fang, M., Boulianne, G.L., and Xie, W. (2011). Neuroligin 2 is required for synapse development and function at the Drosophila neuromuscular junction. J Neurosci 31, 687-699.

Tabuchi, K., and Sudhof, T.C. (2002). Structure and evolution of neurexin genes: insight into the mechanism of alternative splicing. Genomics 79, 849-859.

Tian, Y., Li, T., Sun, M., Wan, D., Li, Q., Li, P., Zhang, Z.C., Han, J., and Xie, W. (2013). Neurexin regulates visual function via mediating retinoid transport to promote rhodopsin maturation. Neuron 77 , 311-322.

Tully, T., and Quinn, W.G. (1985). Classical conditioning and retention in normal and mutant Drosophila melanogaster. J Comp Physiol A, 157, 263-277.

Zeng, X., Sun, M., Liu, L., Chen, F., Wei, L., and Xie, W. (2007). Neurexin-1 is required for synapse formation and larvae associative learning in Drosophila. FEBS Lett 581, 2509-2516.

Open Access This article is distributed under the terms of the Creative Commons Attribution License which permits any use, distribution, and reproduction in any medium, provided the original author(s) and source are credited. 